\section{Efeito da Injeção Percutânea de Etanol na Redução de Nódulos Tiveoideanos}

\section{RESUMO}

Para verificar a eficácia da injeção percutânea de etanol (IPE) no tratamento de nódulos tireoideanos (NT) comparou-se o volume de 86 nódulos (77 pacientes), antes e após uma ou duas sessões de alcoolização. As medianas do volume inicial e final foram respectivamente: $3,5 \mathrm{~mL}(0,3$ a 82,7$)$ e $1,8 \mathrm{~mL}(0$ a 29,4). Sete por cento dos nódulos desapareceram e a média de redução volumétrica foi de 52,6\% $\pm 31,1$ $(p<0,0001)$. Os diferentes padrões ultra-sonográficos responderam distintamente ao tratamento, com as seguintes medianas de redução: $37,3 \%$ (variando de $-39,0$ a 82,6 ) nos NT sólidos; $53,5 \%(14,6$ a 88,0$)$ nos predominantemente sólidos; $58,0 \%(21,0$ a 64,5) nos mistos; $71,0 \%(18,8$ a $100,0)$ nos predominantemente císticos e $90,9 \%(45,9$ a 100,0$)$ nos císticos. Adicionalmente, buscou-se comparar a variação do maior diâmetro dos NT, inicialmente sem tratamento, e após a alcoolização, cuja diferença foi estatisticamente significante $(p<0,00001)$. A complicação mais freqüente foi dor moderada, em $27,6 \%$ dos casos. Concluímos que a IPE mostrou ser uma forma de tratamento segura e eficaz no tratamento de NT benignos. (Arq Bras Endocrinol Metab 2006;50/1:97-104)

Descritores: Nódulo da glândula tireóide; Bócio nodular; Ełanol; Esclerose; Ecografia; Escleroterapia

\begin{abstract}
Percutaneous Ethanol Injection for the Treatment of Thyroid Nodules. To verify the effectiveness of percutaneous ethanol injection (PEI) in the treatment of thyroid nodules (TN), we analyzed 86 TN from 77 patients before and after one or two sessions of PEI. The medians of the initial and final volumes were: $3.5 \mathrm{~mL}$ (range $0.3-82.7$ ) and $1.8 \mathrm{~mL}$ (range 0 - 29.4). Seven percent of the nodules disappeared and the mean nodule size reduction was $52.6 \%$ ( $p<0.0001)$. After ethanol injection, the median volume reduction was: $37.3 \%$ (range -39.0 - 82.6) for solid; $53.5 \%$ (14.688.0) for mostly solid; $58.0 \%$ (21.0 - 64.5) for mixed; $71.0 \%(18.8-100.0)$ for mostly cystic and $90.0 \%$ (45.9 - 100.0) for cystic lesions. Furthermore, we compared the variation of the greatest diameter of TN, without any treatment and after PEl, and found this difference statistically significant $(\mathrm{p}<0.00001)$. Pain was the most common complication, although this was mostly described as moderate (27.6\%). We conclude that PEl is an efficient and safe treatment option for benign TN. (Arq Bras Endocrinol Metab 2006;50/1:97-104)
\end{abstract}

Keywords: Thyroid nodule; Goiter, nodular; Ethanol; Sclerosis; Ultrasonography; Scleroteraphy

$\mathrm{O}$ NÓDULO TIREOIDEANO (NT) BENIGNO é uma condição endócrina freqüente (1) e não existia, até a introdução da IPE, uma forma de tratamento segura e eficaz capaz de promover a sua redução.

\section{artigo original}

Daysi M. de Alcântara-Jones Leila Maria Batista Araújo Alessandro de M. Almeida Daniel de Alcântara Jones Lázaro José Góes Cardoso Marize Carvalho Passos

Curso de Pós-graduação em Medicina e Saúde da

Universidade Federal da Babia (DMAJ, LMBA, AMA); Universidade Severino Sombra, $R J$

(DAJ); e Monte Tabor - Centro Italo-Brasileiro de Promoção Sanitária-Hospital São Rafael, $B A$ (DMAJ, LGC, MP). 
Muitas patologias podem se manifestar clinicamente como um nódulo na tireóide $(2,3)$, mas menos de $10 \%$ deles são malignos. Em razão da grande quantidade de NT presentes na população geral, cerca de 4 a $7 \%$ detectáveis à palpação, e da pequena quantidade dos que são indicados para tratamento cirúrgico, a maioria dos NT constituem um desafio pelos transtornos que podem causar, em razão de fenômenos compressivos locais ou mesmo estéticos.

A injeção percutânea de etanol (IPE) guiada pela ultra-sonografia (USG) já havia sido usada para reduzir massas tumorais hepáticas e abdominais $(4,5)$, além de paratireóides hiperplásicas (6) por alguns autores que, em determinado momento, passaram a utilizá-la no tratamento de NT. No início da década de noventa, Livraghi e cols. (7) registraram a cura clínica de hiperfunção tireoidiana de oito pacientes portadores de nódulos "quentes", autônomos, além de obter significativa redução do volume desses nódulos. A literatura européia, especialmente a italiana, é rica em publicações que ratificam a eficiência desse método no tratamento de NT, quer funcionantes (8-13) ou não (14-16). Lesões císticas parecem responder muito satisfatoriamente ao efeito esclerosante do álcool (17).

Poucas são as publicações fora da Itália (18-20) que registram experiência com a esclerose em NT através da alcoolização e menos de $8 \%$ dos clínicos indicam esse procedimento nos Estados Unidos (21). Assim, o objetivo desse estudo foi avaliar a efetividade da IPE na redução dos NT, após uma ou duas sessões de alcoolização.

\section{CASUÍSTICA E MÉTODOS}

De março de 2001 a junho de 2004 foram selecionados, no Ambulatório do Hospital São Rafael (Salvador, $\mathrm{BA})$, setenta e sete pacientes (4M:73F) portadores de bócios uni-nodulares e multi-nodulares, perfazendo um total de 86 NT tratados. A maioria dos pacientes já tinha indicação de outro profissional para realizar a IPE.

Todos os pacientes foram avaliados clínica e laboratorialmente, com determinações séricas de T3, T4 livre, TSH ultrassensível e anticorpo antitireoperoxidase (anti-TPO). A cintilografia tireoidiana foi indicada quando houve evidência clínica e laboratorial de hiperfunção tireoidiana. Os pacientes não se encontravam em uso de medicação que afetasse a função tireoidiana, exceto em dois casos de hipotireoidismo. Nenhum paciente tinha história prévia de irradiação de cabeça e pescoço. Três pacientes haviam se submetido à cirurgia tireoidiana previamente.
Selecionou-se, para esse trabalho, pacientes portadores de bócio nodular único ou que apresentaram um nódulo em cada lobo ou um nódulo dominante, indiscutivelmente maior que os outros, cuja finalidade foi avaliar a evolução do nódulo e não confundir com outro que não estava sendo tratado. Não foi feita restrição quanto ao tamanho dos NT císticos. Em relação aos nódulos sólidos ou predominantemente sólidos, foram selecionados aqueles com volume menor que $12 \mathrm{~mL}$.

Foram realizadas duas punções aspirativas guiadas pela USG em todos os NT, inclusive nos nódulos cistificados em que se buscou puncionar a sua parte sólida. Foram excluídos do estudo os nódulos que apresentaram à citologia padrão folicular grau II ou superior, segundo classificação proposta na literatura (22) ou padrão de células de Hürthle, e aqueles que foram positivos para malignidade.

A punção aspirativa da tireóide guiada pela USG realizada pela segunda vez confirmou a benignidade do nódulo em todos os pacientes.

Esse estudo foi aprovado pelo Comitê de Ética em Pesquisa do Hospital Universitário Professor Edgard Santos (UFBA) e do Hospital São Rafael (Salvador, BA). Todos os pacientes assinaram o termo de consentimento livre e esclarecido, concordando em participar do estudo.

\section{Estudo ultra-sonográfico}

As USG foram realizadas com aparelho de marca ALOKA, modelo SSD 1700 com "Doppler" colorido, usando-se transdutor de 7,5 MHz. Determinou-se a distância dos pontos mais distantes do centro do NT (sentidos longitudinal, transversal e ântero-posterior); o produto dessas três dimensões foi multiplicado por uma constante $(3,14 / 6)$, estimando-se o volume do NT antes e depois da alcoolização (19).

Dois observadores realizaram o exame ultrasonográfico dos pacientes independentemente, sendo que eles desconheciam o volume anterior do nódulo. Em 30 pacientes, duas mensurações do mesmo NT foram realizadas por um e consecutivamente por outro observador, sem que houvesse comunicação entre eles, a fim de conhecer a variação de suas medições.

Baseadas em suas características ultra-sonográficas, as lesões nodulares foram subdivididas em cinco grupos: sólidas (quando não se visualizava qualquer área de cistificação), predominantemente sólidas (quando o conteúdo líquido não excedia $50 \%$ do volume total do nódulo), predominantemente císticas (quando o conteúdo líquido excedia $50 \%$ do volume do nódulo), císticas (quando a área sólida representava menos 
de $10 \%$ do volume do nódulo) e mistas (quando foi impossível quantificar a parte líquida que se encontrava dispersa pelo conteúdo sólido, em pequenas áreas).

A USG que foi realizada no momento em que se identificou a existência do NT forneceu a primeira dimensão (maior diâmetro) do nódulo, em um primeiro momento. A USG realizada imediatamente antes da IPE forneceu a segunda dimensão do nódulo. Conhecendo-se o tempo decorrido entre essas duas medidas, foi possível avaliar a evolução do NT nesse período e comparar com a alteração do maior diâmetro do NT após a IPE, através de uma USG realizada depois da intervenção.

\section{Técnica da IPE}

A IPE foi realizada no paciente deitado em decúbito dorsal, com um travesseiro sob as regiões escapulares a fim de produzir a hiperextensão do pescoço. Não se utilizou anestésico para realização do procedimento.

Nos NT císticos ou com cistificação, aspirava-se o seu conteúdo líquido, conforme preconizam os autores $(17,23)$, utilizando uma seringa de $10 \mathrm{~mL}$ e agulha de $30 \times 8 \mathrm{~mm}$, tendo o cuidado de deixar parte do líquido na cavidade para não se perder a visualização da agulha. Ao ser obtido o aspirado, desconectava-se a seringa da agulha e desprezava-se o material aspirado. Com a agulha ainda no mesmo local, adaptavase a ela uma seringa contendo álcool absoluto e estéril a $99,8 \%$. Em todos os casos, a intenção foi injetar o etanol na quantidade equivalente a $50 \%$ do volume aspirado (18), no entanto, a aceitação do paciente, naquele momento, foi que determinou a maior ou menor quantidade de álcool efetivamente injetada $(17,18)$. Permanecido na cavidade por quinze minutos, procedia-se a retirada de parte do volume injetado.

Nos NT sólidos ou naqueles em que não havia quase nenhum líquido a ser aspirado, a expectativa era injetar cerca de $20 \%$ do volume do NT, conforme registrado imediatamente antes da IPE. No entanto, também nesses casos, a aceitação do paciente foi o que limitou o volume a ser infundido. Fazia-se a injeção, lentamente, a uma velocidade média de $1 \mathrm{~mL}$ em 10 minutos, até o paciente acusar dor.

O tratamento era considerado concluído quando: 1) o NT desaparecia antes da segunda ou terceira sessão; 2) o NT se apresentava muito pequeno após a última sessão, tornando-se endurecido, não se deixando perfurar pela agulha; 3) o NT sólido não apresentava mais vascularização; 4) o NT mudava completamente sua ecogenicidade; 5) o volume do etanol injetado em uma ou duas sessões era igual ou maior que o volume inicial do NT.
O tratamento foi considerado interrompido quando o procedimento não foi bem aceito por parte do paciente, ou quando houve dificuldade para seu deslocamento até o nosso serviço. Foi tratado um só NT do paciente por sessão.

\section{ANÁLISE ESTATÍSTICA}

As variáveis contínuas foram descritas por média \pm desvio padrão, mediana e respectivas variações, e as variáveis categóricas, como proporções. $\mathrm{O}$ índice de redução do NT foi dado pela razão entre a diferença dos volumes (final - inicial) e o volume inicial, multiplicada por 100 .

Utilizou-se o teste de Wilcoxon para comparar o volume inicial e final dos NT, para comparar os seus maiores diâmetros e também para verificar se a redução obtida com uma sessão foi diferente quando se utilizaram duas sessões. O teste de Friedman comparou simultaneamente as três maiores dimensões dos NT, antes do período de observação, antes da IPE e depois dela. O teste de Kruskal-Wallis foi usado para comparar a redução dos NT dos cinco grupos com diferentes padrões ultra-sonográficos.

O teste de correlação de Spearman avaliou a correlação entre o volume inicial do nódulo e a sua taxa de redução volumétrica, além da correlação do volume de líquido aspirado com a redução dos NT. O método de Bootstrap (24) verificou a significância da diferença entre as medidas dos volumes dos NT, fornecidas pelos observadores, à USG, para um a de $5 \%$.

Os cálculos foram realizados utilizando o programa SPSS (Statistical Package for Social Sciences), versão 11.5 (para Windows) e o Ox 3.30 (Oxford), sendo considerados como estatisticamente significantes valores de $\mathrm{p}$ inferiores a $5 \%(\mathrm{p}<0,05)$.

\section{RESULTADOS}

As características clínicas dos pacientes estudados encontram-se na tabela 1 .

O padrão ultra-sonográfico mais comum dos NT foi o tipo sólido $(n=34,39,5 \%)$, seguido por um padrão predominantemente sólido $(n=21,24,4 \%)$, predominantemente cístico $(\mathrm{n}=18,20,9 \%)$, cístico $(\mathrm{n}=$ $10,11,6 \%)$ e misto $(n=3,3,5 \%)$. Houve variação significativa entre os volumes iniciais dos NT, média \pm DP de 7,6 $\pm 11,5 \mathrm{~mL}$, mediana de $3,5 \mathrm{~mL}(0,3$ a 82,7$)$ e os volumes finais, média de $3,1 \pm 4,5 \mathrm{~mL}$, mediana 
de $1,8 \mathrm{~mL}(0$ a 29,4$)$ e esta foi estatisticamente significante (figura 1 ).

$\mathrm{Na}$ figura 2, observa-se a mediana de redução volumétrica dos NT, estratificados pelas suas características ultra-sonográficas. Pôde-se constatar que quanto maior o conteúdo cístico do nódulo, maior foi a taxa de redução de seu volume $(p<0,0001)$.

A distribuição do etanol foi claramente visualizada durante o procedimento. A área dentro do NT onde era injetado o álcool modificava sua ecogenicidade, passando freqüentemente a ficar com padrão hiperecóico. Em um paciente apresentando NT cístico, o conteúdo líquido espesso dificultou a aspiração. Esse nódulo apresentou uma redução de $45,9 \%$, bem inferior à média dos outros NT císticos (90,8\%).

Quando se procurou analisar a evolução dos NT antes e após a IPE, viu-se que a média \pm DP do maior diâmetro dos NT no início da observação foi de $2,6 \pm 1,0 \mathrm{~cm}$, mediana de $2,5 \mathrm{~cm}(1,0-6,5)$. Após período médio $\pm \mathrm{DP}$ de observação de $8,8 \pm 11,0$ meses, o maior diâmetro do NT foi (média \pm DP) de $2,9 \pm 1,3 \mathrm{~cm}$ e mediana de $2,58(1,0-6,6) \mathrm{cm}$. A diferença entre as medidas foi estatisticamente significante $(p<0,003)$. Realizada a alcoolização em uma ou duas sessões, e após tempo médio \pm DP de 3,0 \pm 2,5 meses, variando de 0,8 a 12 meses, a média do maior diâmetro do NT foi de $2,1 \pm 1,1 \mathrm{~cm}$, mediana

Tabela 1. Características clínicas dos 77 pacientes estudados antes do tratamento. Os valores são expressos em média (idade e tempo de evolução do NT) e em número com respectivos percentuais (para as demais variáveis).

\begin{tabular}{lc}
\hline Característica & Média \pm desvio-padrāo \\
\hline Idade (anos) & $44,8 \pm 16,2$ \\
Tempo de evolução do NT (anos) & $3,4 \pm 4,4$ \\
Sexo & \\
Masculino - N (\%) & $4(5,2)$ \\
Feminino - N (\%) & $73(94,8)$ \\
Função tireoidiana & $64(83,1)$ \\
Normal & $9(11,7)$ \\
T4 e T3 normais com TSH suprimido & $2(2,6)$ \\
Hipotireoidismo & $2(2,6)$ \\
Hipertireoidismo & \\
Anticorpos antitireoideanos & $(5,0) / 4(6,7)$ \\
Positivos - anti-TPO / anti-Tg & $53(88,3)$ \\
Negativos & 17 \\
Não dosados & \\
Cirurgia prévia da tireóide & $3(3,9)$ \\
Sim & $74(96,1)$ \\
Não & \\
Localização do nódulo & $41(47,7)$ \\
Lobo direito & $35(40,7)$ \\
Lobo esquerdo & $6(7,0)$ \\
Istmo & $4(4,7)$ \\
Indefinido & $34(39,5)$ \\
Número de sessões & $52(60,5)$ \\
Uma &
\end{tabular}

$2,2 \mathrm{~cm}(0-5,4)$, sendo que a diferença entre a medida anterior e esta foi significante estatisticamente $(\mathrm{p}<$ $0,0001)$. Assim, o maior diâmetro dos NT desta série teve um crescimento médio de $10,3 \%$ durante a fase de observação e uma redução média de $27,6 \%$ ( $\mathrm{p}<$ 0,00000001 ) após a alcoolização.

A média do volume de etanol injetado foi de $3,64 \pm 5,09 \mathrm{~mL}$. Seis NT $(7,0 \%)$ desapareceram após IPE. Os NT que foram alcoolizados em duas sessões reduziram mais que os que sofreram uma só sessão $(\mathrm{p}<$ $0,0001)$. Não houve correlação entre o volume inicial e o percentual de redução do nódulo $(\mathrm{r}=0,13 ; \mathrm{p}=0,2)$ nem correlação entre o volume aspirado e o percentual de redução do NT $(r=0,29 ; p=0,3)$.

Realizamos em 30 pacientes duas medições dos NTs, efetuadas por dois ultra-sonografistas independentemente, e calculamos a significância da diferença entre elas para um alfa de $5 \%$. A média das diferenças entre as medidas foi de $0,50 \mathrm{~mL}$, e a probabilidade de que uma diferença maior que $0,7 \mathrm{~mL}$ entre duas medidas seja em razão do acaso é menor que $5 \%$.

A limitação de só tratar NT sólidos até $12 \mathrm{~mL}$ objetivou diminuir o erro de medida desses nódulos. NT maiores que $12 \mathrm{~mL}$ não podem ser vistos integralmente no monitor do aparelho de USG e, assim, só se consegue visualizar parte dele. A medida desses nódulos depende da concepção espacial que o

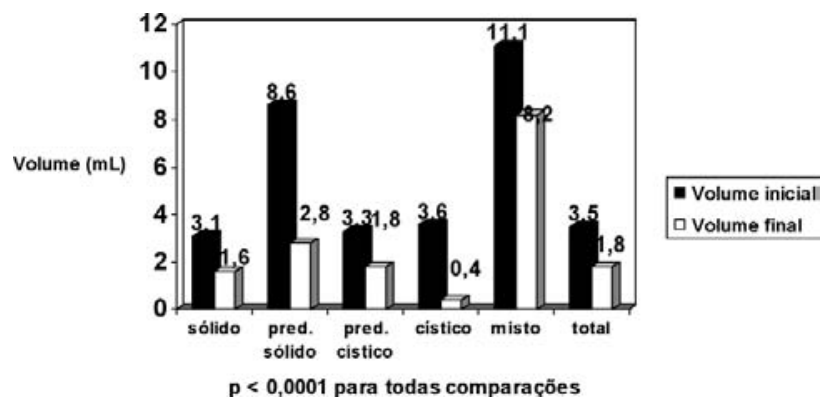

Figura 1. Medianas dos volumes dos NT antes (volume inicial) e após (volume final) as sessões de IPE nos NT sólidos ( $n=$ 34), predominantemente sólidos $(n=21)$, predominantemente císticos $(n=18)$, císticos $(n=10)$ e mistos $(n=3)$.

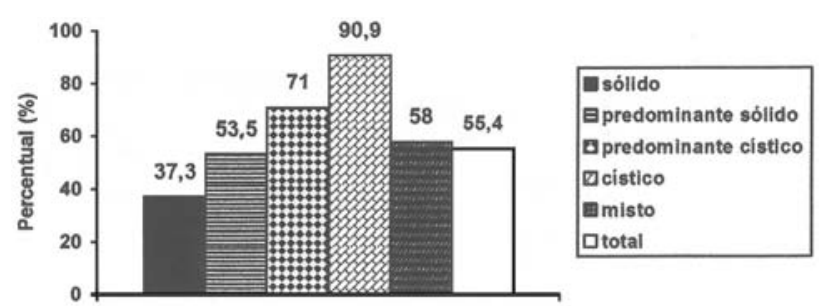

Figura 2. Medianas das taxas de redução dos NT, conforme o padrão da USG. 
observador faz da sua estrutura, causando uma discrepância enorme entre as medidas, até mesmo de um mesmo observador, em momentos diferentes. NT com conteúdo cístico de qualquer tamanho foram aceitos porque, no momento em que o líquido era aspirado, havia uma redução repentina do volume do nódulo e habitualmente a sua mensuração se tornava confiável. A seguir, o volume de líquido aspirado foi somado ao volume da parte sólida.

A figura 3 ilustra o caso de uma paciente de 39 anos, portadora de um nódulo predominantemente cístico, com um formato excêntrico, assemelhava-se a um "oito deitado" que englobava o lobo direito, o istmo e o lobo esquerdo da glândula. $\mathrm{O}$ registro do seu volume ficou sendo a quantidade do líquido aspirado, de 26,6 mL, em razão da discrepância entre os observadores. Após duas sessões, o nódulo reduziu seu volume para $2,4 \mathrm{~mL}$ (redução de $91,1 \%$ ). O caso
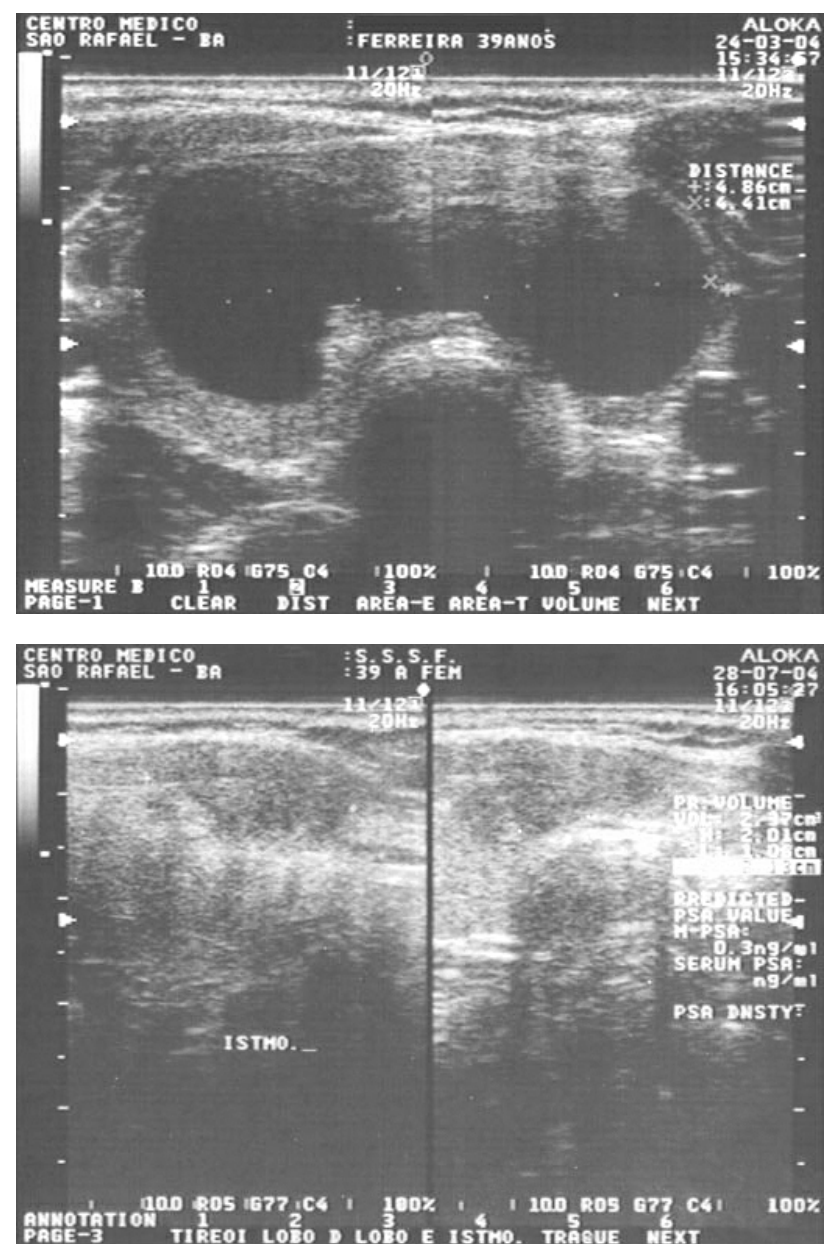

Figura 3. Evolução de NT cístico. Após duas sessões de IPE, o nódulo reduziu de $26,6 \mathrm{~mL}$ para $2,4 \mathrm{~mL}$ (redução de $91,1 \%)$. relatado acima subestima a eficácia do tratamento, mas aumenta o poder do estudo uma vez que reduz o erro tipo 1 (alfa).

O tratamento foi considerado concluído em 27 $(31,4 \%)$ casos, sendo que em $6(7,0 \%)$ os nódulos desapareceram, em $17(19,8 \%)$ tornaram-se muito pequenos para serem puncionados, e em $4(4,7 \%)$ passaram a apresentar um aumento de pressão no nódulo, dificultando a injeção.

O tratamento foi interrompido em $15,1 \%$ dos casos ( 3 pacientes não o suportaram; em 6 pacientes houve redução menor que $15 \%$ do volume inicial após a primeira sessão, desencorajando-nos a prosseguir com o tratamento; uma paciente apresentou disfonia e perdemos o contato com três pacientes). Em 53,5\% das vezes o tratamento não foi considerado concluído, ou porque ainda havia vascularização no interior do nódulo ou porque havia sido injetado etanol numa quantidade inferior ao volume inicial do nódulo. Entretanto, entre os casos com tratamento não concluído, $76,1 \%$ já haviam reduzido mais de $50 \%$ do seu volume inicial.

Nas 138 sessões de alcoolização realizadas, a complicação mais freqüente foi dor $(50,0 \%)$, sendo na maioria das vezes de leve $(16,5 \%)$ e de moderada $(27,6 \%)$ intensidade (tabela 2$)$. A dor freqüentemente se irradiou, geralmente para o ouvido homolateral ou para a arcada dentária, e a maioria dos pacientes referiu que essa só se manifestava no momento da injeção. Ao se interromper a aplicação de etanol, ainda com a agulha em posição, os pacientes autorizavam a continuidade do procedimento, após perceberem que a dor desaparecia tão logo cessava a injeção. Em $15,1 \%$ das vezes os pacientes não referiram qualquer tipo de desconforto. Alguns pacientes usaram antiinflamatórios não hormonais após as sessões de alcoolização. Duas pacientes apresentaram disfonia, que persistiu por uma semana em uma paciente e três semanas em outra, até total resolução.

Tabela 2. Complicações apresentadas em 138 sessões de IPE em NT com número $(n)$ e respectivos percentuais.

\begin{tabular}{lc}
\hline Complicações & $\mathbf{n}(\%)$ \\
\hline Desconforto & $38(25,0)$ \\
Dor leve & $25(16,5)$ \\
Dor moderada & $42(27,6)$ \\
Dor intensa & $9(5,9)$ \\
Calor local & $6(3,3)$ \\
Odinofagia & $3(2,0)$ \\
Disfonia & $2(1,3)$ \\
Febre & $1(0,6)$ \\
Outros* & $3(2,0)$ \\
\hline${ }^{*}$ Edema local cefaléia, medo.
\end{tabular}




\section{DISCUSSÃO}

O percentual de redução volumétrica dos NT desta série não pode ser comparado com o de outras séries, uma vez que não encontramos outro trabalho que estratificasse os nódulos segundo os padrões ultrasonográficos aqui definidos e que tenha usado um número fixo de sessões de alcoolização como foi feito. Também não temos conhecimento de uma série que tenha trabalhado com padrões ultra-sonográficos diferentes, utilizando um número limitado de sessões.

A taxa média de redução volumétrica dos NT desta série foi de $52,6 \%$, que foi superior à redução constatada por Wesche e cols. (25), que usaram iodo radioativo em um grupo de 32 portadores de bócios nodulares (redução de 46\%) em comparação ao grupo que usou doses supressivas de hormônio tireoideano por dois anos (taxa de redução de $22 \%$ ). Ressalte-se que, neste último grupo, $57,1 \%$ dos nódulos não apresentaram qualquer resposta ao tratamento.

Alguns autores $(18,19)$ definiram como critério de "cura" quando havia uma redução maior que 50\% do volume inicial do NT. Nesta série, 78,6\% dos NT císticos (dados não mostrados) tiveram uma resposta similar a esse tratamento. Mais da metade destes $(54,6 \%)$ desapareceu, sendo quatro com uma só sessão e dois nódulos com duas sessões de alcoolização. Nossos achados são similares ao de Bannedbaek e cols., que obtiveram redução de lesões císticas, através da IPE, em $82 \%$ dos 33 pacientes portadores de NT únicos, palpáveis, que se manifestavam com sintomas compressivos, para menos que $1 \mathrm{~mL}$, a maioria dos quais realizou apenas uma sessão de alcoolização (18).

Os NT sólidos do presente estudo não responderam tão rapidamente como as lesões císticas ao poder esclerosante do álcool; entretanto, essa nova forma de tratamento tem se mostrado incomparavelmente superior à supressão com hormônio tireoideano $(15,25)$. Nos NT sólidos desta série ficou evidente a necessidade de mais sessões para considerar o caso concluído.

Este trabalho mostrou que a média de redução dos NT sólidos ou predominantemente sólidos em uma sessão foi de $38,6 \%$ do seu volume inicial. Não existe, na literatura, um consenso de quanto deve ser a redução de um NT sólido entre uma sessão e outra, de modo a se definir se o tratamento deve ser continuado ou não. Parece que quando se consegue infiltrar o NT satisfatoriamente com boa difusão pelo nódulo, observável à USG em uma única punção (agulha na mesma posição sobre a pele), espera-se uma redução de no mínimo $20 \%$ do volume inicial. Se não obtivemos esse percentual, devemos ponderar se deve-se prosseguir com a técnica. Contrapõe-se a essa afirmativa a possibilidade de, numa sessão, atingirmos o vaso que mantém a irrigação daquele nódulo, situação em que há uma redução muito rápida do seu volume. Alguns autores utilizam o Doppler para orientar a IPE, no sentido da maior eficiência do método, fazendo reduzir o NT com um menor número de sessões (10).

Diversos trabalhos que avaliam a redução de NT, quer utilizando a IPE (18), quer utilizando formas convencionais de tratamento, como doses supressivas de hormônio tireoideano $(25,26)$, mostram que NT menores reduzem mais facilmente que os maiores. Nesta série, não foi encontrada correlação entre o índice de redução com o volume inicial do nódulo.

Também não se constatou correlação significativa entre a redução do tamanho do NT e o volume de líquido aspirado nas lesões cistificadas. Essa era uma expectativa dos observadores que freqüentemente verificavam a maior facilidade de redução dos nódulos císticos e cistificados dos quais era aspirada uma grande quantidade de líquido, antes da alcoolização. A explicação para não se ter alcançado uma correlação significativa pode se dever ao fato de termos, em nossa amostra, apenas dez nódulos císticos e possivelmente por termos sido pouco agressivos na aspiração do líquido, antes da alcoolização. Um NT cístico, cujo conteúdo foi tão espesso que não pode ser aspirado, comportou-se à IPE como um nódulo sólido, apresentando uma redução volumétrica bem inferior à redução dos outros cistos.

Possivelmente a maior limitação dos trabalhos que avaliam tratamento de NT por métodos não cirúrgicos se relaciona aos erros de medição desses nódulos, principalmente quando se trabalha com estruturas maiores que $12 \mathrm{~mL}$. Essa limitação é assinalada por alguns autores (27) e nos preocupou desde o início.

A avaliação de 30 NT, cuja medição foi realizada independentemente por dois observadores, procurou minimizar esse erro e, mais que isso, conhecê-lo. Também por essa razão os nódulos sólidos foram limitados a $12 \mathrm{~mL}$. Não foi colocada limitação para os nódulos císticos porque o líquido aspirado permite avaliar o seu volume com maior precisão. $\mathrm{O}$ conhecimento desse desvio permite ainda que se avalie se a redução do nódulo que ocorreu com a última sessão de alcoolização foi significativa ou não. Assim, uma diferença entre o volume anterior e posterior a uma sessão de alcoolização menor que $0,5 \mathrm{~mL}$ pode não significar qualquer variação mas somente a variação de medidas. 
O coeficiente de variação para medidas repetidas de um NT de um mesmo paciente, obtidas em 30 pacientes escolhidos ao acaso, foi de 0,7 . Nesse grupo só foram incluídos seis NT maiores que $10 \mathrm{~mL}$, predominando, portanto, nódulos pequenos, com menor diferença entre os observadores. Essa diferença foi semelhante à obtida por Celani e cols. (28), que encontraram discordância média de $0,5 \pm 0,03 \mathrm{~mL}$ em 40 determinações e menor que a diferença de $11,7 \mathrm{~mL}$ encontrada por Papini e cols., que contabilizaram as medidas de três observadores numa população de 100 pacientes (29).

Constituiu uma limitação desse trabalho a variação de tamanho dos $\mathrm{NT}$, dificuldade que tem sido apresentada por outros autores $(14,21,22)$ que também trabalharam com a heterogeneidade dos NT em relação aos diversos aspectos, inclusive em resposta a esse tipo de tratamento.

Em todos os pacientes encaminhados após avaliação clínica e de imagem (USG e cintilografia) e que tinham uma punção aspirativa com baixa probabilidade de malignidade, a realização de uma segunda biópsia reforçou a provável natureza benigna do nódulo. Esse achado tem sido observado por outros autores $(29,30)$, o que vem confirmar a especificidade desse teste diagnóstico.

A referência de dor como principal complicação dessa técnica tem sido reportada em várias séries e é comum a referência de que ela é transitória, geralmente durando menos de 10 minutos, o que corresponde ao tempo decorrido em uma sessão de esclerose (18). Há relato de autores que utilizaram lidocaína associada ao álcool na esclerose (31) com melhora em relação a essa queixa, sem que isso tenha produzido os mesmos resultados em outras séries (21).

Embora a maioria dos NT permaneça com seu tamanho praticamente inalterado após o diagnóstico (32), é possível que um NT cresça (29), reduza ou mesmo desapareça espontaneamente $(33,34)$ no curso de sua história natural. Tendo em vista essa possibilidade, procurou-se também registrar a variação da maior dimensão do NT (como já registrada por outros autores [33]), quando não se realizou qualquer tipo de intervenção, e comparar com a variação do seu maior diâmetro após a IPE.

Este trabalho mostrou que houve um significativo aumento do maior diâmetro dos nódulos e, contrariamente à sua evolução natural, a IPE produziu uma redução de suas dimensões. No entanto, o tempo variável para se medir o efeito da IPE na redução dos NT pode ter influenciado os nossos resultados, sendo necessário conhecer a evolução dessas lesões após o tratamento, a médio e a longo prazo.
Concluímos que a IPE é um método alternativo de tratamento dos NT, apresentando-se mais eficiente quanto maior é o grau de cistificação das lesões. Quando executado com habilidade, é eficaz e seguro e suas complicações, embora freqüentes, são transitórias e autolimitadas.

\section{REFERÊNCIAS}

1. Mazzaferri EL. Current concepts: Management of a solitary thyroid nodule. N Engl J Med 1993;328:553-9.

2. Murussi M, Pereira CEFN, Brasil BMAA, Tschiedel B. Punção aspirativa da tireóide com agulha fina em um Hospital geral: Estudo de 754 punções. Arq Bras Endocrinol Metab 2001;45:576-83.

3. Lima MA, Yamada AF, Navarro FC, Resende EM, Ferreira B, Borges MF. Punção biópsia aspirativa de tireóide em região endêmica de bócio colóide. Arq Bras Endocrinol Metab 2002;46:275-9

4. Livraghi T, Festi D, Monti F, Salmi A, Vettori C. US-guided percutaneous alcohol injection of small hepatic and abdominal tumors. Radiology 1986;161:309-12.

5. Livraghi T, Salmi A, Bolondi L, Marin G, Arienti V, Monti F, et al. Small hepatocellular carcinoma: Percutaneous alcohol injection-results in 23 patients. Radiology $1988 ; 168: 313-7$.

6. Solbiati L, Giangrande A, De Pra L, Bellotti E, Cantù P, Ravetto $C$. Percutaneous ethanol injection of parathyroid tumors under US guidance: treatment for secondary hyperparathyroidism. Radiology 1985; 155:607-10.

7. Livraghi T, Paracchi A, Ferrari C, Bergonzi M, Garavaglia $G$, Raineri $P$, et al. Treatment of autonomous thyroid nodules with percutaneous ethanol injection: Preliminary results. Radiology 1990; 175:827-9.

8. Goletti O, Monzani F, Caraccio N, Del Guerra P, Lippolis PV, Pucciarelli $\mathrm{M}$, et al. Percutaneous ethanol injection treatment of autonomously functioning single thyroid nodules: Optimization of treatment and short-term outcome. World J Surg 1992; 16:784-90.

9. Livraghi T, Paracchi A, Ferrari C, Reschini E, Macchi RM, Bonifacino A. Treatment of autonomous thyroid nodules with percutaneous ethanol injection: 4-year experience. Radiology 1994; 190:529-33.

10. Lippi F, Ferrari C, Manetti L, Rago T, Santini F, Monzani F, et al. Treatment of solitary autonomous thyroid nodules by percutaneous ethanol injection: Results of an Italian multicenter study. J Clin Endocrinol Metab 1996;81:3261-4.

11. Cerbone G, Spiezia S, Colao A, Marzullo P, Assanti AP, Lucci $R$, et al. Percutaneous ethanol injection under power Doppler ultrasound assistance in the treatment of autonomously functioning thyroid nodules. J Endocrinol Invest 1999:22:752-9.

12. Tarantino L, Giorgio A, Mariniello N, Stefano G, Perrotta A, Aloísio $V$, et al. Percutaneous ethanol injection of large autonomous hyperfunctioning thyroid nodules. Radiology 2000;214:143-8. 
13. Zingrillo $M$, Torlontano $M$, Ghiggi MR, Frusciante V, Varraso A, Liuzzi A, et al. Radioiodine and percutaneous ethanol injection in the treatment of large toxic thyroid nodule: A long-term study. Thyroid 2000; 10:985-9.

14. Bennedbaek FN, Nielsen LK, Hegedüs L. Effect of percutaneous ethanol injection therapy versus suppressive doses of L-thyroxine on benign solitary solid cold thyroid nodules: A randomized trial. J Clin Endocrinol Metab 1998;83:830-5.

15. Goletti O, Monzani F, Lenziardi M, Lippolis PV, De Negri F, Caraccio $N$, et al. Cold thyroid nodules: A new application of percutaneous ethanol injection treatment. J Clin Ultrasound 1994;22:175-8.

16. Zingrillo $M$, Collura D, Ghiggi MR, Nirchio V, Trischitta V. Treatment of large cold benign thyroid nodules not eligible for surgery with percutaneous ethanol injection. J Clin Endocrinol Metab 1998:83:3905-7.

17. Verde G, Papini E, Pacella CM, Gallotti C, Delpiano S, Strada $S$, et al. Ultrasound guided percutaneous ethanol injection in the treatment of cystic thyroid nodules. Clin Endocrinol (Oxf) 1994:41:719-24.

18. Bennedbaek FN, Hegedüs L. Treatment of recurrent thyroid cysts with ethanol: A randomized double-blind controlled trial. J Clin Endocrinol Metab 2003;88:5773-7.

19. Braga-Basaria M, Trippia MA, Stolf AR, Mesa Jr C, Graf H. Tratamento de nódulos autônomos e císticos da tireóide com injeção intranodular de etanol. Rev Assoc Bras 2002;48:335-40.

20. Bianchini EX, Ikejiri ES, Mamone MC, Paiva ER, Maciel RMB, Furlaneto RP. Injeção percutânea de etanol no tratamento de nódulos tireoideanos sólidos, císticos e qutônomos. Arq Bras Endocrinol Metab 2003:47:543-51.

21. Bonnema SJ, Bennedbaek FN, Ladenson PW, Hegedüs L. Management of the nontoxic multinodular goiter: a North American survey. J Clin Endocrinol Metab 2002;87:112-7.

22. Camargo RYA, Tomimori EK. Diagnóstico dos nódulos tireoideos baseado na avaliação ultra-sonográfica e citológica combinada. Arq Bras Endocrinol Metab 1998:42:273-6

23. Iacconi P, Spinelli C, Monzani F, Miccoli P. Percutaneous ethanol injection for thyroid cysts: A Word for caution. Clin Endocrinol (Oxf) 1996:44:126.

24. Efron B, Tibshirani R J. An introduction to the Bootstrap. Chapman \& Hall / CRC, 1993.
25. Wesche MFT, Tiel-v Buul MMC, Lips P, Smits NJ, Wiersinga WM. A randomized trial comparing L-thyroxine with radioactive iodine in the treatment of sporadic nontoxic goiter. J Clin Endocrinol Metab 2001;86:998-1005.

26. Lima N, Knobel M, Cavaliere H, Sztejnsznajd C, Tomimori E, Medeiros-Neto G. Levothyroxine suppressive therapy is partially effective in treating patients with benign solid thyroid nodules and multinodular goiters. Thyroid 1997:7:691-7

27. Zelmanovitz F, Genro S, Gross JL. Suppressive therapy with levothyroxine for solitary thyroid nodules: A doubleblind controlled clinical study and cumulative metaanalyses. J Clin Endocrinol Metab 1998;83:3881-5.

28. Celani MF. Levothyroxine suppressive therapy in the medical management of nontoxic benign multinodular goiter. Exp Clin Endocrinol 1993; 101:326-32.

29. Papini E, Petrucci L, Guglielmi R, Panunzi C, Rinaldi R, Bacci V, et al. Long-term changes in nodular goiter: A 5year prospective randomized trial of levothyroxine suppressive therapy for benign cold thyroid nodules. J Clin Endocrinol Metab 1998;83:780-3

30. Brito DH, Graf H, Collaço LM. Valor da repunção aspirativa na doenca nodular benigna da tireóide. Ara Bras Endocrinol Metab 2001:45:246-51.

31. Papini E, Panunzi C, Pacella CM, Bizzarri G, Fabbrini R, Psicchio $G$, et al. Percutaneous ultrasound-guided ethanol injection: A new treatment of toxic autonomously functioning thyroid nodules? J Clin Endocrinol Metab 1993;76:411-6.

32. Samuels MH. Editorial: Evaluation and treatment of sporadic nontoxic goiter - some answers and more questions. J Clin Endocrinol Metab 2001:86:994-7.

33. Cheung PSY, Lee JMH, Boey JH. Thyroxine suppressive therapy of benign solitary thyroid nodules: A prospective randomized study. World J Surg 1989:13:818-21.

34. Kuma K, Matsuzuka F, Kobayashi A, Hirai K, Morita S, Miyauchi $A$, et al. Outcome of long standing solitary thyroid nodules. World J Surg 1992;16:583-7.

\section{Endereço para correspondência:}

Daysi Maria de Alcântara-Jones

Loteamento Jardim do Atlântico, Quadra C, Lote 19

427000-000 Lauro de Freitas, BA

E-mail: daysijones@terra.com.br 